Pfizer and Lilly, Guillermo Suárez Amorín: None declared, Patricia Setien Preciados: None declared, M. Cristina Mata Arnaiz: None declared, Miguel Á. González-Gay Grant/research support from: AbbVie, MSD and Roche, Speakers bureau: AbbVie, MSD and Roche, Ricardo Blanco Grant/research support from: Abbvie, MSD and Roche, Consultant of: Abbvie, Pfizer, Roche, Bristol-Myers, Janssen and MSD, Speakers bureau: Abbvie, Pfizer, Roche, Bristol-Myers, Janssen, Lilly and MSD

DOI: 10.1136/annrheumdis-2020-eular.5005

\section{AB0459 MORTALITY OF RHEUMATOID VASCULITIS AGGRAVATED BY CLINICALLY LATENT TUBERCULOSIS - A RETROSPECTIVE CLINICOPATHOLOGIC STUDY OF 161 AUTOPSY PATIENTS}

Á. Apáthy ${ }^{1}$, M. Bély ${ }^{2}{ }^{1}$ St. Margaret Clinic, Department of Rheumatology, Budapest, Hungary; ${ }^{2}$ Hospital of the Order of the Brothers of Saint John of God, Department of Pathology, Budapest, Hungary

Background: Systemic vasculitis of autoimmune origin (e.g. rheumatoid vasculitis - RV) plays a pivotal role in the pathogenesis of rheumatoid arthritis (RA) [1]. $\mathrm{RV}$ may be characterized by non-specific inflammation (nsRV) with or without fibrinoid necrosis (fnRV) or by granulomatous transformation of blood vessels (grRV) [1].

The prevalence and mortality of tuberculosis (TB) is higher in rheumatoid arthritis (RA) than in the general population.

Objectives: The aim of this study was to assess the influence of dormant (inactive), clinically latentTB (with or without subclinical atypical miliary exacerbation) on the mortality of rheumatoid vasculisit (RV).

Methods: At the National Institute of Rheumatology 9475 patients died between 1969 and 1992; among them 161 with RA and all of them were autopsied [1].

RA was confirmed clinically according to the criteria of the ARA.

nsRV alone or in combination with fnRV or grRV was diagnosed at autopsy, and confirmed by a detailed review of extensive histological material [1].

The prevalence fibrous (fTB), fibrocaseous (fcTB) or miliary TB (mTB) was diagnosed and characterized histologically, reviewing retrospectively the clinical and pathological reports [1].

From each patient a total of 50-100 tissue blocks of 12 organs (heart, lung, liver, spleen, kidneys, pancreas, gastrointestinal tract, adrenal glands, skeletal muscle, peripheral nerve, skin and brain) were studied microscopically.

The possible role of fTB, fcTB or mTB on the mortality due to nsRV, fnRV or grRV was analyzed with Pearson's chi-squared $\left(x^{2}\right)$ test.

Results: nsRV complicated RA in $\mathbf{3 3}(\mathbf{2 0 . 4 9 \% )}$ ) 161 patients, in combination with fnRV in $\mathbf{1 7}(\mathbf{5 1 . 5 1} \%)$ or with grRV in $\mathbf{1 0}(\mathbf{3 0 . 3 0} \%)$ of 33 cases.

The nsRV led to death in $\mathbf{1 9}(\mathbf{5 7 . 5 7} \%)$ of 33 patients, in combination with fnRV in $11(64.71 \%$ of 17$)$ or with $\mathbf{g r R V}$ in $\mathbf{7}(\mathbf{7 0 . 0} \%$ of 10$)$ cases; the mortality due to nsRV, fnRV or grRV showed an increasing tendency.
Post-primary TB was associated with RA in 21 (13.04\%) of 161 patients, 12 (57.14\% of 21$)$ were fTB, and 9 ( $42.86 \%$ of 21$)$ fcTB. One of 12 fTB and 5 of 9 fcTB were complicated with discrete miliary dissemination (mTB) in $6(3.7 \%$ of $161 ; 28.57 \%$ of 21$)$ RA patients.

TB $(n=21)$ was associated with fatal $n s R V$ in $\mathbf{5}$, with fnRV in $\mathbf{3}$, with $\mathbf{g r R V}$ in $\mathbf{3}$ of 21 cases.

The fTB $(n=12)$ was associated with fatal $n s R V$ in 1 , with fnRV in 1 , with grTB in 1 of 12 cases.

The fcTB $(n=9)$ was associated with fatal $n s R V$ in $\mathbf{4}$, with fnRV in $\mathbf{3}$, with grTB in 3 of 9 cases.

mTB $(n=6)$ was associated with fatal nsRV in $\mathbf{3}$, with fnRV in $\mathbf{2}$, with grTB in $\mathbf{3}$ of 6 cases.

There was a significant and positive correlation between fcTB and mortality of nsRV (c: $0.76, x^{2}=9.7593, p<0.0017$ ), between fcTB and mortality of fnRV (c: $0.80, x^{2}=6.5701, p<0.0103$ ), between fcTB and mortality of grRV (c: 0.90 $\left.x^{2}=12.5835, p<0.00038\right)$, The correlation was also positive and significant between $\mathbf{m T B}$ and mortality of $n s R V\left(c: 0.79, x^{2}=5.3406, p<0.0208\right.$ ), between mTB and mortality of grRV (c: $0.95, x^{2}=20.8706, p<0.0000049$ ),

The relationships were not significant between TB or fTB and mortality of nsRV, fnRV or grRV, nor between mTB and mortality of fnRV.

Conclusion: Recognition of indolent TB is a great challenge especially before starting immunosuppressive or biological therapy for RA.

TB may lead directly to death by arrosion of pulmonary arteries with massive pulmonary bleeding, by hematogenous miliary dissemination (Landouzy typhobacillos) or by canalicular spread as a cavernous chronic process (phthisis tuberculosa), etc. Discrete miliary dissemination may be also lethal caused by circulatory failure [1].

The contribution of TB to the mortality of complications of RA or of allied disorders is plausible and can never be excluded.

According to our data the risk of mortality due to $\mathbf{n s R V}$, fnRV or $\mathbf{g r R V}$ showed an increasing tendency, and fnRV or grRV should be regarded the most severe forms of nsRV in RA

The positive and significant correlation between fcTB or MTB and mortality of nsRV, fnRV or grRV represents a positive contribution to the fatal outcome due to $n s R V$, fnRV or grRV

References:

[1] Bély M, Apáthy Á: "Clinical pathology of rheumatoid arthritis." Akadémia Kiadó, Budapest (2012):1-144. http://www.akkrt.hu ISBN 9789630593397 Disclosure of Interests: None declared

DOI: 10.1136/annrheumdis-2020-eular.467

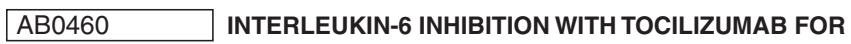 THE TREATMENT OF GIANT CELL ARTERITIS AND POLYMYALGIA RHEUMATICA IN PATIENTS WITH SERIOUS COMORBIDITIES}

T. Beketova ${ }^{1}$, E. Otteva ${ }^{2}$, E. Nasonov ${ }^{1}{ }^{1}$ V.A. Nasonova Research Institute of Rheumatology, Moscow, Russian Federation; ${ }^{2} 2$ Institute for HCW Postgraduate

TABLE.

\begin{tabular}{|c|c|c|c|c|c|c|c|c|c|c|c|}
\hline $\begin{array}{l}\text { CLÍNICAL } \\
\text { PHENOTYPES }\end{array}$ & Cases N (\%) & $\mathrm{COLCH}$ & $\cos$ & Dosis total IS & AZA & MTX & CYA & MMF & TLD & APR & DAP \\
\hline Oral ulcers & $110(99.1)$ & 85 (77.9) & $81(73.6)$ & $51(46.4)$ & $30(27.3)$ & $25(22.7)$ & $14(12.7)$ & $2(1.8)$ & $6(5.5)$ & $6(5.5)$ & $3(2.7)$ \\
\hline Genital ulcers & 69 (62.2) & 56 (81.2) & $51(74)$ & $32(46.4)$ & $17(24.6)$ & $16(23.2)$ & $10(14.5)$ & $1(1.5)$ & $5(7.2)$ & $2(3.4)$ & $3(4.3)$ \\
\hline Cutaneous lesions & $76(68.5)$ & $58(76.3)$ & $61(80.3)$ & $52(68.4)$ & $32(42.1)$ & $22(29)$ & $9(11.8)$ & 0 & $5(6.6)$ & $6(7.9)$ & $3(4)$ \\
\hline Ocular manifestations & $39(35.1)$ & $27(69.2)$ & $36(92.3)$ & $19(48.7)$ & $17(43.6)$ & $12(30.8)$ & $11(28.2)$ & $2(5.1)$ & $4(10.3)$ & $2(5.1)$ & $2(5.1)$ \\
\hline Neurological involvement & $20(18)$ & $12(60)$ & $15(75)$ & $15(75)$ & $3(15)$ & $4(20)$ & $3(15)$ & $1(5)$ & 0 & 0 & $1(5)$ \\
\hline Vascular manifestations & $11(10)$ & $8(72.7)$ & $9(81.8)$ & $5(45.5)$ & $3(27.3)$ & $3(27.3)$ & $1(9.1)$ & 0 & 0 & 0 & 0 \\
\hline Gastrointestinal involvement & $4(3.6)$ & $2(50)$ & $1(25)$ & $1(25)$ & $1(25)$ & 0 & 0 & 0 & 0 & 0 & 0 \\
\hline TOTAL & 111 & $85(76.6)$ & $85(76.6)$ & $51(46)$ & $30(27)$ & $25(22.5)$ & $14(12.6)$ & $2(1.8)$ & $6(5.4)$ & $6(5.4)$ & $3(2.7)$ \\
\hline
\end{tabular}

TABLE 2.

\begin{tabular}{|c|c|c|c|c|c|c|c|c|}
\hline $\begin{array}{l}\text { CLINICAL } \\
\text { PHENOTYPES }\end{array}$ & BT & ADA & IFX & ETN & TCZ & No improvement & Partial improvement & Complete response \\
\hline Oral ulcers & $28(35.5)$ & $22(20)$ & $12(11)$ & $3(2.7)$ & $2(1.8)$ & $22(20)$ & $22(20)$ & $66(60)$ \\
\hline Genital ulcers & $17(24.7)$ & $13(18.8)$ & $8(11.6)$ & $2(2.9)$ & $1(1.4)$ & $16(23.2)$ & $12(17.4)$ & $41(59.4)$ \\
\hline Cutaneous lesions & $21(27.6)$ & $18(23.7)$ & $8(10.5)$ & $3(4)$ & $2(2.6)$ & $8(10.5)$ & $19(25)$ & $49(64.5)$ \\
\hline Ocular manifestations & $19(50)$ & $16(42.1)$ & $9(23.7)$ & $1(2.6)$ & $2(5.3)$ & 0 & $8(21)$ & $30(79)$ \\
\hline Neurological involvement & $7(35)$ & $2(10)$ & $4(20)$ & $1(5)$ & 0 & $3(15)$ & $5(25)$ & $12(60)$ \\
\hline Vascular manifestations & $4(36.4)$ & $3(27.3)$ & $2(18.2)$ & $1(9.1)$ & $1(9.1)$ & $2(18.2)$ & $4(36.4)$ & $5(45.5)$ \\
\hline Gastrointestinal involvement & 0 & 0 & 0 & 0 & 0 & $1(25)$ & $1(25)$ & $2(50)$ \\
\hline TOTAL & $28(25.2)$ & $22(19.8)$ & $12(10.8)$ & $3(2.7)$ & $2(1.8)$ & $22(19.8)$ & $22(19.8)$ & $67(60.4)$ \\
\hline
\end{tabular}

Abbreviations: COLCH: Colchicine; OCS: Oral Corticosteroids; IS: Immunosuppressants; AZA: Azathioprine; MTX: Methotrexate; CYA: Cyclosporine A; MMF: Mycophenolate Mofetil; TLD: Talidomide; APR: Apremilast; DAP: Dapsone; BT: Biologic Therapy; ADA: Adalimumab; IFX: Infliximab; ETN: Etanercept; TCZ: Tocilizumab 
Training, Prof. S.I. Sergeyev Regional Clinical Hospital N1, Khabarovsk, Russian Federation

Background: Giant cell arteritis (GCA) and polymyalgia rheumatica (PMR) are closely related inflammatory conditions affecting people aged over 50 years. Objectives: We present our experience of using tocilizumab (TCZ) therapy for management of GCA/PMR aggravated by severe concurrent pathologies that potentially increase the risk of side effects of glucocorticoids (GCs).

Methods: 22 patients were recruited into the prospective study: six patients with GCA, 13- PMR, and three- with both GCA and PMR, 95.5\% were females, mean age $72.8 \pm 6.5$ years. Mean disease duration was $3.5(0.5-19)$ months. All patients had active GCA/PMR with mean CRP $30.3 \pm 32.7 \mathrm{mg} / \mathrm{l}$. Seven patients had visual ischemic complications, and another one- aortitis. All patients had serious comorbidities, $59 \%$ of patients had three and more severe concurrent diseases. All patients were administered TCZ i/v 2.3-8.8 mg/kg Q4W. 50\% patients were also treated with prednisone at mean 20 (10-70) mg/day. The follow-up period was 24 (6-60) months.

Results: All patients demonstrated good clinical response to TCZ i/v 2.3-8.8 mg/ kg Q4W given for average 4,5 (2-11) months, achieving remission in $100 \%$ of cases. Some patients showed a very rapid improvement after initiation of treatment, including TCZ monotherapy. Prednisone dose was discontinued in 6/11, or was reduced to $2.5(2.5-10) \mathrm{mg}$ in $4 / 11$. There was one relapse after TCZ discontinuation, although this patient managed to regain the remission after resumption of TCZ i/v $4 \mathrm{mg} / \mathrm{kg}$. There was one (4.6\%) serious complication (septic olecranon bursitis 1 month after TCZ discontinuation), one patient died of myocardial infarction 12 months later after TCZ discontinuation. Three remaining complications included one case of peripheral artery disease (claudication), one- psoriasis, and one- sural lipodermatosclerosis.

Conclusion: Interleukin- 6 inhibitors should be considered as potentially effective and relatively safe treatment for GCA/PMR patients with serious comorbidities, intolerance or contraindications to standard therapy. More data is necessary to identify the optimal dosing regimen and duration of TCZ therapy, as well as cost-effectiveness aspects.

Disclosure of Interests: None declared

DOI: 10.1136/annrheumdis-2020-eular.2179

\section{AB0461 $\quad$ ANCA-ASSOCIATED VASCULITIS: CLINICAL FEATURES, RELAPSE, ORGAN DAMAGE AND SURVIVAL IN 197 PATIENTS}

M. Bektas ${ }^{1}$, B. Ince ${ }^{1}$, B. F. Agargun ${ }^{2}$, D. Y. Guzey ${ }^{3}$, Y. Yalçınkaya ${ }^{1}$, B. ArtimEsen $^{1}$, A. Gül ${ }^{1}$, M. L. Ocal ${ }^{1}$, M. Inanc ${ }^{1} .{ }^{1}$ Istanbul Faculty of Medicine, Internal Medicine, Rheumatology Division, Istanbul, Turkey; ${ }^{2}$ stanbul Faculty of Medicine, Internal Medicine, Istanbul, Turkey; ${ }^{3}$ Istanbul Faculty of Medicine, Istanbul, Turkey

Background: ANCA-associated vasculitis (AAV) is a multisystemic autoimmune disease with high mortality and morbidity.

Objectives: We aimed to present the long-term follow-up results of our cohort. Methods: Data of patients who fulfilled Chapell Hill Consensus Criteria and followed up at least 6 months between 1999-2019 were analyzed. A standard form including vasculitis damage index (VDI) was used. Multivariable analysis was performed by using logistic regression.

Results: Long-term data was available for 197 patients (\%53.8 female) from 208 patient records. Mean age at diagnosis was 49.4 years and mean follow-up was 80.7 months. Granulomatosis with polyangiitis (GPA); microscopic polyangiitis (MPA), eosinophilic GPA (EGPA) were $117(64.5 \%), 52(26.4 \%), 17(8.6 \%)$, respectively. Relapses are observed in $31.6 \%$ of patients. Disease relapses were higher in GPA compared to MPA and EGPA $(p=0.014)$. Relapse rate was higher in patients with s.aureus carriage $(\mathrm{p}=0.037)$. Cyclophosphamide (CYC) $(76.6 \%)$ was most commonly used drug for induction, whereas azathioprine $(57.3 \%)$ was used mostly in maintenance. In multivariate analysis relapse was found to be associated with maintenance treatment with rituximab $(p<0.001)$, venous thrombosis $(p=0.046)$ and serious infection $(p<0.004)$. There was no significant association between relapse and mortality. Five-year survival rates were 98.5\% for GPA, $88.5 \%$ for MPA and $100 \%$ for EGPA. Nineteen patients died during follow-up (9.6\%). In univariate analysis mortality were high in MPA patients. Low hemoglobin and increased creatinine at baseline, subglottic stenosis, polyneuropathy, and cerebrovascular events (CVE) were associated with increased mortality. In multivariable analysis, mortality was associated with CVE $(p=0.047)$ and anti-MPO positivity $(\mathrm{p}=0.014)$. Malignancy was developed in 9 patients $(\mathrm{M}$ / F: 7/2; two lung, three bladder, one cervix, one thyroid papillary, one kidney and one of unknown primary). There was no association between malignancy and cumulative dose of CYC. Venous thromboembolism was developed in 12 $(6 \%)$ and avascular necrosis (AVN) was detected in 30 patients (15.4\%). Most (88.7\%) patients developed damage during follow-up. Mean VDI score was 2.6 and VDI score was found to be higher in GPA $(p=0.035)$. There was no association between VDI score and mortality.
Conclusion: In our AAV cohort, GPA was most frequent. Although survival was improved, permanent organ damage was detected in the majority of patients Relapse and organ damage were found to be increased in patients with GPA Relapses are frequent and maintenance with rituximab could not prevent relapses. Also relapses were associated with venous thrombosis and severe infections. Patients should be screened for malignancies especially of the genitourinary tract.

Table 2. Damage findings of AAV patients according to VDI

\begin{tabular}{lc}
\hline Organ/system & Number(\%) \\
\hline Steroid myopathy & $23(\% 11.7)$ \\
Osteoporosis & $31(\% 15.9)$ \\
AVN & $30(\% 15.4)$ \\
Cataract & $30(\% 15.4)$ \\
Partial loss of vision & $6(\% 3.1)$ \\
Blindness (one eye) & $2(\% 1)$ \\
Subglottic stenosis & $9(\% 4.5)$ \\
Hearing loss & $18(\% 9.1)$ \\
Nasal septum perforation & $21(\% 10,7)$ \\
Chronic nasal crusting & $9(\% 4,6)$ \\
Chronic ashtma & $28(\% 14,2)$ \\
Chronic dispnea & $1(\% 0,5)$ \\
Hypertension & $60(\% 30,5)$ \\
Coronary artery disease / Angioplasty & $10(\% 5,1)$ \\
Cardiomyopathy & $6(\% 3)$ \\
Valvular heart disease & $5(\% 2,5)$ \\
Myocardial infarction & $7(\% 3,6)$ \\
Deep vein thrombosis & $12(\% 6)$ \\
Chronic renal failure (GFR $<50 \mathrm{ml} / \mathrm{min})$ & $51(\% 26)$ \\
End stage renal disease & $22(\% 10.8)$ \\
Cerebrovascular accident & $9(\% 4,4)$ \\
Peripheric neuropathy & $39(\% 19.8)$ \\
Malignancy & $9(\% 4.5)$ \\
Diabetes mellitus & $24(\% 12.2)$ \\
Gonadal failure & $2(\% 1)$ \\
\hline &
\end{tabular}

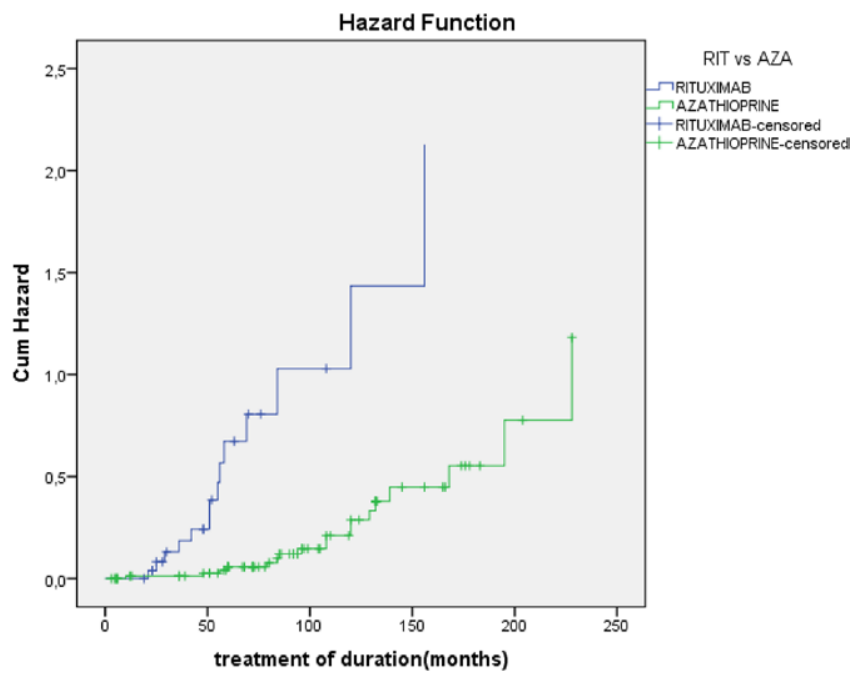

Figure 3. Cumulative Relapse Rate: Hazard ratio of patients treated with Rituximab versus Azathioprine (Log Rank: $p<0.001$

Disclosure of Interests: None declared

DOI: 10.1136/annrheumdis-2020-eular.3899

\section{AB0462 BEHCET'S DISEASE: CLINICAL FEATURES AND OFF- LABEL BIOLOGIC TREATMENT STRATEGIES}

E. Bellis ${ }^{1}$, S. Monti ${ }^{1,2}$, S. Balduzzi ${ }^{1}$, P. Delvino ${ }^{1}$, A. Biglia $^{1}$, C. Montecucco ${ }^{1}$ ${ }^{1}$ IRCCS Policlinico S. Matteo Foundation, University of Pavia, Rheumatology, Pavia, Italy; ${ }^{2}$ University of Pavia, PhD in Experimental Medicine, Pavia, Italy

Background: The treatment of Behçet's disease (BD) is still mainly based on the evidence derived from case reports, case series, retrospective analyses, and few clinical trials suggesting the safety and potential efficacy of off-label use of biologic agents in refractory cases. ${ }^{1}$

Objectives: To describe clinical manifestations and their management, with particular focus on treatment indications, outcomes and safety of biologic therapy, in a cohort of patients with BD. 